\title{
Efficacy of a 14-coil for Framing in Original Coil Embolization of Small Article Cerebral Aneurysms
}

Tetsuhiro Higashida, Takanori Uchida, Manabu Osakabe, Yuichi Takahashi, and Ryuzaburo Kanazawa

Objective: The purpose of this study was to demonstrate the efficacy of a 14-coil (Target $X L$ ) for framing in coil embolization of small cerebral aneurysms.

Methods: Between January 2017 and December 2018, 46 patients underwent coil embolization of a small cerebral aneurysm that was less than $5 \mathrm{~mm}$ in maximum diameter. They were categorized into 26 patients in whom only 10-coils were used and 20 in whom Target XL was used for framing. The volume embolization rate (VER) and recanalization rate were compared between the two groups.

Results: Although there were two patients in whom Target XL was replaced with a 10-coil for framing, no adverse events associated with the use of Target $X L$ were noted. The mean VER of the first framing coil was significantly higher in aneurysms that were framed with Target $X L$ than in those framed with a 10-coil (Target $X L 22.6 \pm 4.5 \%, 10$-coil $17.9 \pm 8.4 \% ; p=0.03$ ). Furthermore, the mean VER at the end of the procedure was significantly higher in aneurysms with Target $\mathrm{XL}$ used for framing than in those embolized using only 10-coils (14-coil: $36.8 \pm 7.8 \%, 10$-coil: $32.0 \pm 6.5 \% ; p=0.03$ ). No recanalization was observed in aneurysms for which Target $\mathrm{XL}$ was used for framing, whereas five aneurysms embolized using only 10-coils were recanalized.

Conclusion: Target XL may be safe and feasible as a framing coil in coil embolization of small cerebral aneurysms, which may result in a high VER, low recanalization rate, and good outcome.

Keywords > 14-coil, framing, small cerebral aneurysm, volume embolization rate, recanalization rate

\section{Introduction}

To reduce the recanalization rate after coil embolization for cerebral aneurysms, the achievement of a high volume embolization rate (VER) is important. ${ }^{1,2}$ Coils with a large primary diameter, which are highly stable in the aneurysm and have a large volume, are considered to be advantageous for increasing the VER. In recent years, highly flexible 14-coils (0.014 inches in the primary diameter), such as Target XL (Stryker, Fremont, CA, USA), have also been

Department of Neurosurgery, Nagareyama Central Hospital, Nagareyama, Chiba, Japan

Received: February 3, 2020; Accepted: April 24, 2020

Corresponding author: Tetsuhiro Higashida. Department of Neurosurgery, Nagareyama Central Hospital, 2-132-2, Higashihatsuishi, Nagareyama, Chiba 270-0114, Japan

Email: tets018@gmail.com

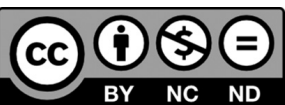

This work is licensed under a Creative Commons Attribution-NonCommercialNoDerivatives International License.

(C)2020 The Japanese Society for Neuroendovascular Therapy used for small cerebral aneurysms. In general, 10-coils ( 0.010 inches in the primary diameter) are used in coil embolization of small cerebral aneurysms. However, as the use of 14-coils for framing based on a careful decision can safely improve the embolization efficiency, we have actively performed framing using Target XL for small aneurysms ( $<5 \mathrm{~mm}$ in diameter). In this study, framing using a 10-coil and Target XL were compared regarding the VER and recanalization rate in coil embolization of small cerebral aneurysms, and the efficacy of framing with Target XL was evaluated.

\section{Materials and Methods}

On 3D reconstruction images of rotational cerebral angiography, the dimensions of aneurysms (height, length, and width) were measured, and aneurysms with a maximum diameter $<5 \mathrm{~mm}$ were defined as small aneurysms. Cases after 2017 when framing with Target XL was initiated in our institution were evaluated. Between January 2017 and December 2018, 46 small aneurysms (46 patients) were 
treated by coil embolization. The 46 patients were classified into 26 who underwent framing using a 10-coil (10-coil group) and 20 who underwent framing using Target XL (Target XL group), and were retrospectively analyzed.

In the 10-coil group, the coils used for framing were Target (Stryker), Hydrosoft 10, Hydroframe 10 (Terumo, Tokyo, Japan), Penumbra Smart Coil (Penumbra, Alameda, CA, USA), and Axium Prime (Medtronic Minneapolis, MN, USA). In the Target XL group, Target XL was used for only framing as the first coil. The coil type and adjunctive technique were selected based on the aneurysm shape and the presence of rupture.

Follow-up cerebral angiography was performed 6-12 months after surgery. In addition, MRI was performed at 3-6-month intervals. Cerebral angiography was also performed whenever aneurysmal recanalization is suspected by MRA. As a parameter of the embolization state for cerebral angiography, the Raymond score (RS) was used (RS1, complete obliteration; RS2, residual neck; and RS3, residual aneurysm). ${ }^{3)}$ Using the RS, aggravation of the aneurysm embolization state compared with that immediately after treatment was defined as recanalization.

The aneurysm volume, coil volume, VER, VER using the first coil (1st VER), and VER at the completion of coil embolization (final VER) were calculated as follows:

$$
\begin{aligned}
& \text { Aneurysm volume }=\text { height } \times \text { length } \times \text { width } \times \frac{\pi}{6} \\
& \text { Coil volume }=(\text { primary diameter })^{2} \times \text { length } \times \frac{\pi}{4} \\
& 1 \text { st VER }(\%)=\frac{\text { volume of the } 1 \text { st coil }}{\text { aneurysm volume }} \times 100 \\
& \text { Final VER }(\%)=\frac{\text { all coil volume }}{\text { aneurysm volume }} \times 100
\end{aligned}
$$

The patient background (age and sex), aneurysm factors (location, maximum diameter, and presence of rupture), treatment factors (stent use and number of coils), 1st VER, final VER, embolization state immediately after treatment (RS), and the presence of recanalization were compared between the two groups.

For statistical analysis, the unpaired t-test was used for continuous variables and $\chi^{2}$ test for nominal variables, and $\mathrm{p}<0.05$ was regarded as significant.

\section{Results}

The mean age of the 46 patients ( 37 females, $80.4 \%$ ) was $63.1 \pm 13.0$ years. There were 19 patients $(41.3 \%)$ with a ruptured aneurysm. The aneurysm was located in the internal carotid artery in 25 patients, anterior cerebral artery including the anterior communicating artery in 12 , middle cerebral artery in 6 , and vertebrobasilar artery in 3 . The mean maximum aneurysm diameter was $3.7 \pm 0.7 \mathrm{~mm}$ and the mean minimum diameter was $2.9 \pm 0.7 \mathrm{~mm}$. The mean number of used coils was $3.6 \pm 1.8$, and a stent was used in 13 patients. These basic data in the 10-coil and Target XL groups are shown in Tables 1 and 2. The maximum and minimum aneurysm diameters were both larger in the Target XL group, and a significant difference was observed in the minimum diameter $(p=0.04)$. Ruptured aneurysms were significantly more frequently observed in the 10-coil group ( $\mathrm{p}=0.02$ ), and a stent was slightly more frequently used in the Target XL group $(\mathrm{p}=0.06)$. The 10-coil group included two patients in whom a 10-coil was used for framing because satisfactory framing was unable to be performed using Target XL. There were no adverse events associated with the use of Target XL.

The results of coil embolization and postoperative course in the two groups are shown in Table 3 . The mean 1st VER was significantly higher $(\mathrm{p}=0.03)$ in the Target XL group $(22.6 \pm 4.5 \%)$ than in the 10-coil group $(17.9 \pm 8.4 \%)$. The mean final VER was also significantly higher $(\mathrm{p}=0.03)$ in the Target XL group (36.8 \pm $7.8 \%)$ than in the 10 -coil group $(32.0 \pm 6.5 \%)$. At the completion of coil embolization, the percentage of patients exhibiting a favorable embolization state (RS 1) did not differ between the 10-coil group (17/26 patients, 65\%) and the Target XL group (14/20 patients, $70 \%$ ). However, during a mean follow-up period of 24.9 months, clear aneurysm recanalization was noted in five patients in the 10-coil group, but in none in the Target XL group $(p=0.05)$. The patients who demonstrated recanalization are shown in Table 4 . In these patients, a ruptured aneurysm was frequently observed $(p=0.1)$ and no stent was used $(p=0.3)$. However, neither difference was significant.

\section{Case Presentation (Fig. 1)}

A 58-year-old female presented with sudden headache and disturbed consciousness, and was diagnosed with subarachnoid hemorrhage (World Federation of Neurosurgical Societies [WFNS] grade 2, Fisher grade 2). Coil embolization of an irregular-shaped right middle cerebral aneurysm $(3.4 \times 2.6 \times$ $4.1 \mathrm{~mm}$ ) was performed. A 6-Fr Launcher (Medtronic, Minneapolis, MN, USA) and a 4.2-Fr FUBUKI (Asahi Intecc, Tokyo, Japan) were coaxially advanced, and placed in the 
Table 1 Characteristics of 10-coil group and Target XL group

\begin{tabular}{lccc} 
& $\begin{array}{c}10 \text {-coil } \\
(\mathrm{n}=26)\end{array}$ & $\begin{array}{c}\text { Target XL } \\
(\mathrm{n}=20)\end{array}$ & p value \\
\hline Age (years) & $63.0 \pm 15.2$ & $63.2 \pm 9.6$ & 0.9 \\
Female & $20(76 \%)$ & $17(85 \%)$ & 0.7 \\
Ruptured aneurysm & $15(57 \%)$ & $4(20 \%)$ & 0.02 \\
Maximum aneurysm diameter $(\mathrm{mm})$ & $1.6-4.8$ & $3.0-4.9$ & 0.3 \\
Mean & $3.6 \pm 0.8$ & $3.8 \pm 0.5$ & \\
Median & 3.8 & 3.9 & \\
Minimum aneurysm diameter $(\mathrm{mm})$ & $1.5-4.2$ & $2.6-4.0$ & 0.04 \\
Mean & $2.8 \pm 0.8$ & $3.1 \pm 0.4$ & \\
Median & 2.7 & 3.2 & \\
Stent use & $4(15 \%)$ & $9(45 \%)$ & 0.06 \\
Number of coils & $3.3 \pm 1.8$ & $4.0 \pm 1.7$ & 0.2 \\
\hline
\end{tabular}

Table 2 Aneurysm location

\begin{tabular}{lcc} 
& 10-coil $(n=26)$ & Target XL $(n=20)$ \\
ICA & 14 & 11 \\
A1 segment of ACA & 1 & 0 \\
Acom & 5 & 2 \\
Distal ACA & 1 & 3 \\
MCA & 3 & 3 \\
VA-BA & 2 & 1 \\
\hline ACA: anterior cerebral artery; Acom: anterior communicating artery; BA: basilar artery; ICA: internal \\
carotid artery; MCA: middle cerebral artery; VA: vertebral artery
\end{tabular}

Table 3 Comparison of results between 10-coil cases and Target XL cases

\begin{tabular}{lccl} 
& $\begin{array}{c}10 \text {-coil } \\
(\mathrm{n}=26)\end{array}$ & $\begin{array}{c}\text { Target XL } \\
(\mathrm{n}=20)\end{array}$ & p value \\
Mean 1st VER (\%) & $17.9 \pm 8.4$ & $22.6 \pm 4.5$ & 0.03 \\
Mean final VER (\%) & $32.0 \pm 6.5$ & $36.8 \pm 7.8$ & 0.03 \\
Complete obliteration (RS 1) at coiling & $17(65 \%)$ & $14(70 \%)$ & 0.9 \\
Recanalization at follow-up & $5(19 \%)$ & $0(0 \%)$ & 0.05 \\
Mean follow-up period (months) & $25.1 \pm 8.2$ & $24.6 \pm 8.3$ & 0.8 \\
\hline
\end{tabular}

RS: Raymond score; VER: volume embolization rate

right internal carotid artery, and an SL-10 (Stryker) was navigated into the aneurysm. Framing was performed using Target XL 360 Soft $2 \times 6$, which was followed by insertion of Target nano $3601.5 \times 3$ and Target nano $3601 \times 2$, and Axium Prime Helical $1 \times 2$. Satisfactory embolization was achieved (RS1). The 1st VER using Target XL was 31.4\% and the final VER was $44.8 \%$. Her postoperative course was favorable and she was discharged home 32 days after the operation with a modified RS of 0 . Cerebral angiography 1 year after the operation demonstrated no aneurysm recanalization and a maintained favorable embolization state.

\section{Discussion}

In general, coil embolization yields more favorable outcomes than clipping because of fewer neurological complications associated with surgical manipulation, but it has a high recanalization rate. ${ }^{4,5}$ Besides ruptured, large, or wide-neck aneurysms are known to be related to high recanalization rate after the coil embolization, a low VER was confirmed to increase the recanalization rate. ${ }^{1,2,6}$ To reduce the recanalization rate, coils modified with hydrogel or bioabsorbable polymers have been developed, and hydrogel has been reported to have specific effects. ${ }^{7-9)}$ The concept of hydrogel is to increase the VER by hydrogel expansion within the aneurysm. Thus, to prevent recanalization, the achievement of a high VER is important. ${ }^{2,6)}$

To achieve a high VER, flexible coils with differing shapes have been developed in order for aneurysms to be packed as densely as possible. ${ }^{10-12)}$ However, favorable framing in the aneurysm in the initial stage of embolization 


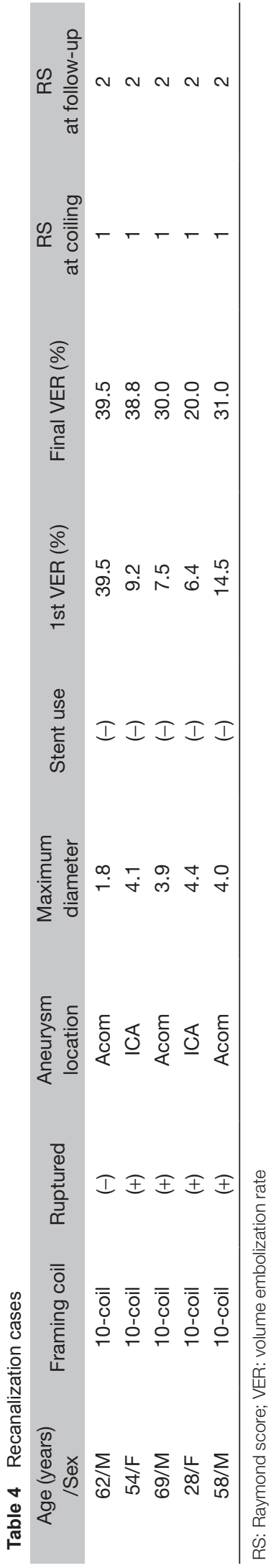

is the most important factor to achieve favorable results of coil embolization. When a favorable frame is formed, the hemodynamics within the aneurysms change, not only promoting aneurysm thrombosis but also enabling safe and efficient embolization to achieve a high VER. Thus, good framing is directly associated with the success of surgery and treatment results. ${ }^{13,14)}$ Coils used for framing should have both high stability, sufficiently covering the aneurysm neck without collapse, and flexibility to conform to different aneurysm shapes, being uniformly distributed in the aneurysm. The achievement of a high VER using the first coil has been reported to improve the final VER; therefore, coil selection for framing is important. ${ }^{6,15-17)}$ Relatively long coils with a complex shape are frequently used for framing, and coils with a large primary diameter are expected to achieve a high 1st VER.

In terms of the achievement of a high VER, 18-coils (primary diameter, 0.018 inches) have been reported to improve the VER. ${ }^{11,18-21)}$ However, 18-coils are not usable for microcatheters with a tip internal diameter $<0.018$ inches that are generally used for coil embolization of small aneurysms while 14-coils can be used for such microcatheters. As their volume is approximately twice that of 10-coils with the same length, framing using a 14-coil is expected to achieve a high 1st VER. In addition, as 14-coils are thicker than 10-coils, firm stable frames that rarely collapse during insertion of 10-coils into the frame can be formed, which enables safe coil embolization and the achievement of a high final VER. ${ }^{22)}$

In this study, Target XL mini series with a secondary diameter $<5 \mathrm{~mm}$ were used. Target XL mini have a primary diameter of 0.014 inches, and are the thickest coils among those with a secondary diameter $<5 \mathrm{~mm}$ that are used at present in Japan. Target XL mini are softer than Target XL with a secondary diameter $\geq 5 \mathrm{~mm}$, and are safe when used for small aneurysms, including ruptured aneurysms. In addition, these coils have a complex shape and high flexibility, and can conform to the wall of aneurysms with a relatively irregular shape without forming compartments. As the area of contact with the aneurysm wall is large due to the coil thickness, a good stable frame uniformly covering the entire aneurysm can be readily formed. The actual feeling of use is soft, and microcatheter kickback during coil insertion is relatively rare. Coil embolization for small aneurysms has a high risk of hemorrhagic complications associated with aneurysmal perforation, and careful attention is particularly necessary for ruptured aneurysms. ${ }^{23)}$ There is concern that a possible increase in the risk of 

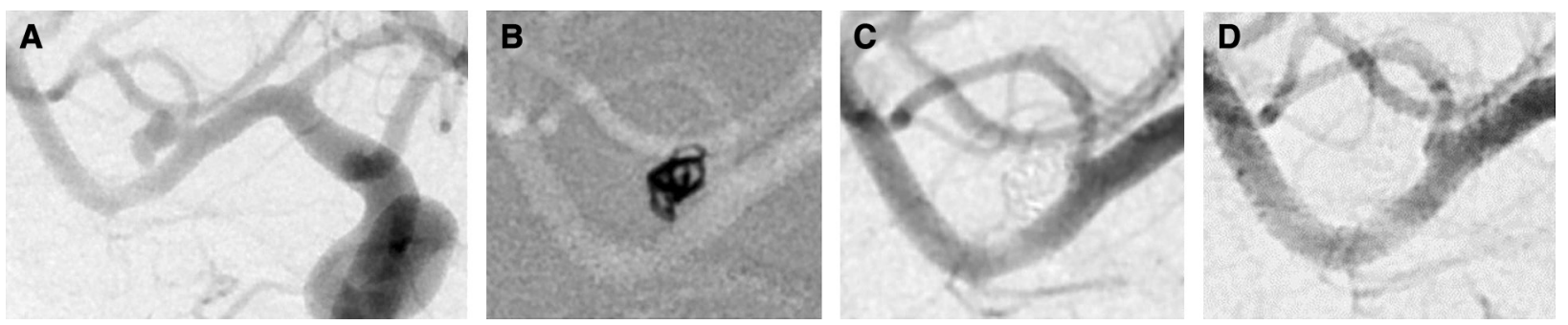

Fig. 1 A 58-year-old woman who presented with subarachnoid hemorrhage. (A) An irregular-shaped aneurysm, which was $3.4 \times 2.6 \times 4.1$ $\mathrm{mm}$ in size, was located at the bifurcation of the right middle cerebral artery. (B) Target XL $2 \times 6$ was used for framing. Note that the coil was uniformly fit to the irregular-shaped aneurysmal wall. (C) Complete obliteration of the aneurysm was achieved at the end of the procedure. (D) No notable recanalization was observed on follow-up angiography 1 year after coil embolization of the aneurysm

aneurysmal perforation due to the use of thick coils, but no adverse events due to the use of Target XL was observed, although there were a few patients in whom Target XL was changed to a 10-coil for framing. As described above, Target XL is excellent in flexibility and operability, and can also be safely used for small aneurysms within a reasonable range for appropriately selected cases.

Concerning the selection of the Target XL size, as a high VER can be achieved using one Target XL coil for small aneurysms, a coil size slightly smaller in the secondary diameter than the diameter of the aneurysm is recommended for accurate coil placement within the aneurysm. At our institution, there are no definite criteria regarding the minimum diameter of aneurysms for the use of Target $\mathrm{XL}$. However, as the smallest secondary diameter of Target $\mathrm{XL}$ is $2 \mathrm{~mm}$, the use of Target XL for aneurysms $\leq 2.5 \mathrm{~mm}$ in diameter may be difficult, considering the above cautions for size regarding accurate coil placement within the aneurysm. Indeed, the smallest minimum diameter of aneurysms for which Target XL was used was $2.6 \mathrm{~mm}$ in this study. On the other hand, 10-coils include those with a secondary diameter $<2 \mathrm{~mm}$ and can be readily used for aneurysms $\leq 2.5 \mathrm{~mm}$ in diameter. In this study, only the 10 -coils were used for aneurysms $\leq 2.5 \mathrm{~mm}$ in diameter. Concerning the size of aneurysms, the difference in the minimum aneurysm diameter was more marked than that in its maximum diameter between the Target $\mathrm{XL}$ and 10-coil groups. This may have been because the use of Target XL was considered to be inappropriate for aneurysms with a particularly small diameter, and patients with such aneurysms were allocated to the 10-coil group.

In this study, framing with Target XL in coil embolization for small cerebral aneurysms with a maximum diameter $<5 \mathrm{~mm}$ resulted in a high final VER. For a long period after the coil embolization, a stable embolization state was maintained. Although the use of thick coils has been reported to reduce the number of coils and be economical, the number of coils used in the Target XL group was not smaller than that in the 10-coil group in this study. ${ }^{18,19,24)}$ The reason for the lack of reduction in the number of coils in the Target XL group may have been because Target XL was used only for framing, and more coils were able to be inserted due to the stable frame of Target XL and the stent assistance in some patients. However, as a result, a high final VER was achieved, which is more important in treatment than a reduction in the number of coils.

This study has several limitations; therefore, it cannot be concluded that only the use of Target XL for framing led to the reduction in recanalization cases. This study was retrospective and there were no definite criteria for the use of Target XL. The decision on whether Target XL was used was left to the operator or physician in charge. In addition, as there were some patients in whom framing with Target $\mathrm{XL}$ was impossible, and the coil was changed to a 10-coil, aneurysms for which Target XL was difficult to use were allocated to the 10-coil group, which is a selection bias. Thus, aneurysms for which Target XL is readily used may have been originally unlikely to be recanalized even using 10-coils alone, and many aneurysms that were unlikely to be recanalized might be included in the Target XL group. In particular, many unruptured aneurysms and many aneurysms treated by stent-assisted coil embolization may have been major factors associated with the low recanalization rate. The following reasons can be considered for the infrequent use of Target XL for ruptured aneurysms. There was reluctance to insert thick coils into small ruptured aneurysms and difficulty in using a stent in the acute stage of ruptured aneurysms. In addition, thick coils were considered to be inappropriate for ruptured aneurysms with an irregular shape. In this study, the recanalization rate in all 46 patients was $10 \%$ (5/46 patients). Recanalization was observed only in the 10-coil group, and 
the recanalization rate in this group was 19\% (5/26 patients), which is slightly higher than the previously reported recanalization rate of aneurysms $<5 \mathrm{~mm}$ in diameter $(9-18 \%){ }^{23)}$ However, it should be noted that all patients with recanalization in this study demonstrated a change from RS1 to RS2 after embolization, and none had RS3. In addition, of the 5 patients with recanalization, 4 had ruptured aneurysms and none underwent stentassisted coil embolization. The recanalization rate of ruptured aneurysms was reported to be high, ${ }^{6,25)}$ and the use of a stent was reported to reduce the recanalization rate. ${ }^{26)}$ There is a possibility that many ruptured aneurysms and aneurysms treated without a stent that are often recanalized were allocated to the 10-coil group, which increased the recanalization rate in this group and exaggerated the superiority of the Target XL group. To evaluate the effectiveness of the use of Target XL compared with other factors for reducing aneurysm recanalization, multivariate analysis is desirable. In this study, there were only a few recanalization cases and multivariate analysis was inappropriate. In the future, to clarify the usefulness of Target $\mathrm{XL}$ and the conditions of aneurysms appropriate for their use, cases should be increased for multivariate analysis and prospective studies should be performed to reduce the selection bias.

This study demonstrated that 14-coils (Target XL) can also be used for some small aneurysms for which thick coils are generally difficult to use. Also, there is a possibility that the use of 14-coils in such small aneurysms can prevent recanalization. Although several types of 14-coils are available for embolization of cerebral aneurysms, Target XL may particularly be suitable for small aneurysms because of the high flexibility and the many options of small secondary diameter.

\section{Conclusion}

Target XL can be safely used as a framing coil in coil embolization of small cerebral aneurysms. Due to a firm stable frame formed by Target XL, there is a possibility that the embolization efficiency improves, resulting in a high final VER. This may lead to a reduction in the aneurysm recanalization rate, thereby leading to favorable treatment results.

\section{Disclosure Statement}

The authors declare no conflict of interest.

\section{References}

1) Slob MJ, Sluzewski M, van Rooij WJ: The relation between packing and reopening in coiled intracranial aneurysms: a prospective study. Neuroradiology 2005; 47: 942-945.

2) Leng B, Zheng Y, Ren J, et al: Endovascular treatment of intracranial aneurysms with detachable coils: correlation between aneurysm volume, packing, and angiographic recurrence. J Neurointerv Surg 2014; 6: 595-599.

3) Mascitelli JR, Moyle H, Oermann EK, et al: An update to the Raymond-Roy occlusion classification of intracranial aneurysms treated with coil embolization. J Neurointerv Surg 2015; 7: 496-502.

4) Ries T, Groden C: Endovascular treatment of intracranial aneurysms: long-term stability, risk factors for recurrences, retreatment and follow-up. Klin Neuroradiol 2009; 19: 62-72.

5) Li H, Pan R, Wang H, et al: Clipping versus coiling for ruptured intracranial aneurysms: a systematic review and meta-analysis. Stroke 2013; 44: 29-37.

6) Neki H, Kohyama S, Otsuka T, et al: Optimal first coil selection to avoid aneurysmal recanalization in endovascular intracranial aneurysmal coiling. J Neurointerv Surg 2018; 10: 50-54.

7) Rezek I, Mousan G, Wang Z, et al: Coil type does not affect angiographic follow-up outcomes of cerebral aneurysm coiling: a systematic review and meta-analysis. AJNR Am J Neuroradiol 2013; 34: 1769-1773.

8) Molyneux AJ, Clarke A, Sneade M, et al: Cerecyte coil trial: angiographic outcomes of a prospective randomized trial comparing endovascular coiling of cerebral aneurysms with either cerecyte or bare platinum coils. Stroke 2012; 43 : 2544-2550.

9) Taschner CA, Chapot R, Costalat V, et al: Second-generation hydrogel coils for the endovascular treatment of intracranial aneurysms: a randomized controlled trial. Stroke 2018; 49: 667-674.

10) Slob MJ, van Rooij WJ, Sluzewski M: Coil thickness and packing of cerebral aneurysms: a comparative study of two types of coils. AJNR Am J Neuroradiol 2005; 26: 901-903.

11) Sluzewski M, van Rooij WJ: Packing performance of helical Guglielmi detachable coil (GDC) 18 in intracranial aneurysms: a comparison with helical GDC 10 coils and complex trufill/orbit coils. AJNR Am J Neuroradiol 2007; 28: $1384-1387$.

12) Wakhloo AK, Gounis MJ, Sandhu JS, et al: Complex-shaped platinum coils for brain aneurysms: higher packing density, improved biomechanical stability, and midterm angiographic outcome. AJNR Am J Neuroradiol 2007; 28: 1395-1400.

13) Schirmer CM, Malek AM: Critical influence of framing coil orientation on intra-aneurysmal and neck region 
hemodynamics in a sidewall aneurysm model. Neurosurgery 2010; 67: 1692-1702; discussion 1702.

14) Khatri R, Chaudhry SA, Rodriguez GJ, et al: Frequency and factors associated with unsuccessful lead (first) coil placement in patients undergoing coil embolization of intracranial aneurysms. Neurosurgery 2013; 72: 452-458; discussion 458.

15) Ishida $W$, Sato $M$, Amano $T$, et al: The significant impact of framing coils on long-term outcomes in endovascular coiling for intracranial aneurysms: how to select an appropriate framing coil. J Neurosurg 2016; 125: 705-712.

16) Misaki K, Uchiyama N, Nambu I, et al: Optimizing the volume of the initial framing coil to facilitate tight packing of intracranial aneurysms. World Neurosurg 2016; 90: 397-402.

17) Sousa EB, Brandão LFS, Tavares CB, et al: Importance of the first coil in the embolization of intracranial aneurysms: a case control study. Clin Neuroradiol 2019; 29: 733-740.

18) Milburn J, Pansara AL, Vidal G, et al: Initial experience using the Penumbra coil 400: comparison of aneurysm packing, cost effectiveness, and coil efficiency. J Neurointerv Surg 2014; 6: 121-124.

19) Mascitelli JR, Patel AB, Polykarpou MF, et al: Analysis of early angiographic outcome using unique large diameter coils in comparison with standard coils in the embolization of cerebral aneurysms: a retrospective review. J Neurointerv Surg 2015; 7: 126-130.
20) Kulcsár Z, Wanke I, Rüfenacht D, et al: Safety and effectiveness of large volume coils in the treatment of small aneurysms. J Neurointerv Surg 2016; 8: 1260-1263.

21) Kaesmacher J, Müller-Leisse C, Huber T, et al: Volume versus standard coils in the treatment of intracranial aneurysms. J Neurointerv Surg 2016; 8: 1034-1040.

22) Imai $T$, Ohshima $T$, Goto $S$ : Comparison of clinical outcomes using 10 coil versus 14 coil in endovascular coil embolization for small and medium-sized intracranial aneurysms: efficacy of 14 coil. JNET J Neuroendovasc Ther 2016; 10: 196-200.

23) Jindal G, Miller T, Iyohe $M$, et al: Small intracranial aneurysm treatment using target ${ }^{\circledR}$ ultrasoft ${ }^{\mathrm{TM}}$ coils. J Vasc Interv Neurol 2016; 9: 46-51.

24) Zhang K, Wang ZL, Gao BL, et al: Use of a first largesized coil versus conventional coils for embolization of cerebral aneurysms: effects on packing density, coil length, and durable occlusion. World Neurosurg 2019; 127: e685-e691.

25) Tan IY, Agid RF, Willinsky RA: Recanalization rates after endovascular coil embolization in a cohort of matched ruptured and unruptured cerebral aneurysms. Interv Neuroradiol 2011; 17: 27-35.

26) Feng MT, Wen WL, Feng ZZ, et al: Endovascular embolization of intracranial aneurysms: to use stent(s) or not? Systematic review and meta-analysis. World Neurosurg 2016; 93: 271-278. 